\title{
A Class of Unbounded Fourier Multipliers on the Unit Complex Ball
}

\author{
Pengtao Li, ${ }^{1}$ Jianhao Lv, $^{2}$ and Tao Qian ${ }^{2}$ \\ ${ }^{1}$ Department of Mathematics, Shantou University, Shantou, Guangdong 515063, China \\ ${ }^{2}$ Faculty of Science and Technology, University of Macau, Macau \\ Correspondence should be addressed to Pengtao Li; li_ptao@163.com
}

Received 5 December 2013; Accepted 15 January 2014; Published 24 February 2014

Academic Editor: Anna Mercaldo

Copyright (c) 2014 Pengtao Li et al. This is an open access article distributed under the Creative Commons Attribution License, which permits unrestricted use, distribution, and reproduction in any medium, provided the original work is properly cited.

We introduce a class of Fourier multiplier operators $M_{b}$ on $n$-complex unit sphere, where the symbol $b \in H^{s}\left(S_{\omega}\right)$. We obtained the Sobolev boundedness of $M_{b}$. Our result implies that the operators $M_{b}$ take a role of fractional differential operators on $\partial \mathbb{B}$.

\section{Introduction}

In this paper, we introduce a class of unbounded holomorphic Fourier multipliers $M_{b}$ on $n$-complex unit sphere. We further study the boundedness of $M_{b}$ on Sobolev spaces. Our results generalize the theory of Fourier multipliers on Lipschitz curves in $\mathbb{C}$ to $n$-complex unit sphere $\mathbb{B}_{n}$. We refer the reader to Gaudry et al. [1], McIntosh and Qian [2], and Qian [3, 4] for further information on multipliers on Lipschitz curves.

Our motivation originates from the following example on the unit sphere in $\mathbb{C}^{n}$. The explicit formula of the CauchySzegö kernel is as follows:

$$
H(z, \bar{\xi})=\frac{1}{\omega_{2 n-1}} \frac{1}{\left(1-z \bar{\xi}^{\prime}\right)^{n}} .
$$

Let $\left\{p_{k}^{v}\right\}$ denote the orthonormal system in the space of holomorphic functions in $\mathbb{B}_{n}$. The following result is wellknown:

$$
H(z, \bar{\xi})=\sum_{k=0}^{\infty} \sum_{v=1}^{N_{k}} p_{v}^{k}(z) \overline{p_{v}^{k}(\xi)}, \quad z \in \mathbb{B}_{n}, \quad \xi \in \partial \mathbb{B}_{n} .
$$

See Theorem 1 and (16) below for details. Formally, (2) can be seen as the special case of (4) below. Let $S_{\omega}$ be the sector defined as

$$
S_{\omega}=\{z \in \mathbb{C}: z \neq 0,|\arg z|<\omega\} .
$$

Assume that

(1) $b$ is holomorphic on $S_{\omega}$;

(2) $b$ is bounded near the origin;

(3) $|b(z)| \leq C|z|^{s}$ for $|z|>1$.

We consider the function:

$$
H_{b}(z, \bar{\xi})=\sum_{k=1}^{\infty} b(k) \sum_{v=1}^{N_{k}} p_{v}^{k}(z) \overline{p_{v}^{k}(\xi)}
$$

If $b(z) \equiv 1$, then (4) becomes (2). For $s=0$, Cowling and Qian [5] introduced a class of bounded holomorphic multipliers on $L^{2}\left(\partial \mathbb{B}_{n}\right)$. In this paper, we consider the case $s \neq 0$. For this case, $b$ is unbounded on $\{z:|z|>1\}$. We prove that if $b \in H^{s}\left(S_{\omega}\right)$, then

$$
\left|H_{b}(z, \bar{\xi})\right|=\frac{C_{\mu^{\prime}}}{\delta\left(\nu, \mu^{\prime}\right)\left|1-z \overline{\xi^{\prime}}\right|^{n+s}} .
$$

See Theorem 5.

In Section 4, we introduce a class of Fourier multipliers $M_{b}$ with $b \in H^{s}\left(S_{\omega}\right), s \neq 0$. Unlike the ones of Cowling and Qian [5], our multipliers $b$ are unbounded on $S_{\omega}$. Take $b(k)=$ $k^{s}$. Plancherel's theorem implies that $M_{b}$ is not bounded on $L^{2}\left(\partial \mathbb{B}_{n}\right)$. Hence for such $M_{b}$, we need to consider their boundedness on some function spaces with higher regularity. 
Let $r, s \in[0, \infty)$. We prove that if $b \in H^{s}\left(S_{\omega}\right), M_{b}$ is bounded from Sobolev space $W^{p, r+s}\left(\partial \mathbb{B}_{n}\right)$ to Sobolev space $W^{p, r}\left(\partial \mathbb{B}_{n}\right)$, $1<p<\infty$. Our result implies that the operators $M_{b}$ take a role of fractional differential operators on $\partial \mathbb{B}_{n}$. See Theorem 11.

The rest of this paper is organized as follows. In Section 2, we state some basic preliminaries and notations which will be used in the sequel. In Section 3, we estimate the kernels generated by holomorphic multipliers $b \in H^{s}\left(S_{\omega}\right)$. The Sobolev boundedness of the operators $M_{b}$ is given in Section 4.

Notations. $\mathrm{U} \approx \mathrm{V}$ represents that there is a constant $c>0$ such that $c^{-1} \mathrm{~V} \leq \mathrm{U} \leq c \mathrm{~V}$ whose right inequality is also written as $\mathrm{U} \lesssim \mathrm{V}$. Similarly, one writes $\mathrm{V} \gtrsim \mathrm{U}$ for $\mathrm{V} \geq c \mathrm{U}$.

\section{Preliminaries and Notations}

In this section, we state some preliminaries and notations and refer the reader to Gong [6], Hua [7], and Rudin [8] for further information. We use $z$ as a general element of $\mathbb{C}^{n}$; that is, $z=\left(z_{1}, \ldots, z_{n}\right), z_{i} \in \mathbb{C}, i=1,2, \ldots, n, n \geq 2$. Denote $\bar{z}=\left[\overline{z_{1}}, \ldots, \overline{z_{n}}\right]$. The notation $z$ is considered to be a row vector. Denote by $\mathbb{B}_{n}$ the open unit ball $\left\{z \in \mathbb{C}^{n}:|z|<1\right\}$, where $|z|=\left(\sum_{i=1}^{n}\left|z_{i}\right|^{2}\right)^{1 / 2}$. The unit sphere in $\mathbb{C}^{n}$ is denoted by

$$
\partial \mathbb{B}_{n}=\mathbb{S}^{2 n-1}=\left\{z \in \mathbb{C}^{n}:|z|=1\right\} .
$$

The open ball centered at $z$ with radius $r$ will be denoted by $B(z, r)$. A general element on $\partial \mathbb{B}_{n}$ is usually denoted by $\xi$. The constant $\omega_{2 n-1}$ involved in the Cauchy-Szegö kernel is the surface area of $\partial \mathbb{B}_{n}$ and is equal to $2 \pi^{n} / \Gamma(n)$. For $z, w \in \mathbb{C}^{n}$, we use the notation $z w^{\prime}=\sum_{k=1}^{n} z_{k} w_{k}$. The theory developed in this paper is relevant to the radial Dirac operator

$$
D=\sum_{k=1}^{n} z_{k} \frac{\partial}{\partial z_{k}}
$$

Now we state some basis knowledge of basic functions in the space of holomorphic function in $\mathbb{B}_{n}$ and some relevant function spaces on $\partial \mathbb{B}_{n}$. We refer to Hua [7] for details. Let $k$ be a nonnegative integer. We consider the column vector $z^{[k]}$ with components

$$
\sqrt{\frac{k !}{k_{1} ! \cdots k_{n} !}} z_{1}^{k_{1}} \cdots z_{n}^{k_{n}}, \quad k_{1}+\cdots k_{n}=k .
$$

The dimension of $z^{[k]}$ is

$$
N_{k}=\frac{1}{k !} n(n+1) \cdot(n+k-1)=C_{n+k-1}^{k} .
$$

Let $d z$ and $d \sigma(\xi)$ be the Lebesgue volume element of $\mathbb{C}^{n}$ and the Lebesgue area element of $\partial \mathbb{B}_{n}$, respectively. Define

$$
\begin{gathered}
H_{1}^{k}=\int_{\mathbb{B}_{n}} \overline{z^{[k]^{\prime}}} \cdot z^{[k]} d z, \\
H_{2}^{k}=\int_{\partial \mathbb{B}_{n}} \overline{\xi^{[k]^{\prime}}} \cdot \xi^{[k]} d \sigma(\xi) .
\end{gathered}
$$

It is easy to prove that $H_{1}^{k}$ and $H_{2}^{k}$ are positive definite Hermitian matrices of order $N_{k}$. There exists a matrix $\Gamma$ such that

$$
\overline{\Gamma^{\prime}} \cdot H_{1}^{k} \cdot \Gamma=\Lambda, \quad \overline{\Gamma^{\prime}} \cdot H_{2}^{k} \cdot \Gamma=I,
$$

where $\Lambda=\left[\beta_{1}^{k}, \ldots, \beta_{n}^{k}\right]$ is a diagonal matrix and $I$ is the identity matrix. Set

$$
\begin{aligned}
& z_{[k]}=z^{[k]} \cdot \Gamma ; \\
& \xi_{[k]}=\xi^{[k]} \cdot \Gamma .
\end{aligned}
$$

Denote by $p_{v}^{k}(z)$ the components of the vectors $z_{[k]}$. From (11), we can see that

$$
\begin{aligned}
& \int_{\mathbb{B}_{n}} p_{v}^{k}(z) \overline{p_{\mu}^{k}(z)} d z=\delta_{v \mu} \cdot \delta_{k l} \cdot \beta_{v}^{k}, \\
& \int_{\partial \mathbb{B}_{n}} p_{v}^{k}(\xi) \overline{p_{\mu}^{k}(\xi)} d \sigma(\xi)=\delta_{v \mu} \cdot \delta_{k l} .
\end{aligned}
$$

The following theorem is well known.

Theorem 1. The system of functions

$$
\left\{\left(\beta_{\nu}^{k}\right)^{-\frac{1}{2}} p_{\nu}^{k}, k=0,1,2, \ldots, v=1,2, \ldots, N_{k}\right\}
$$

is a complete orthonormal system in the space of holomorphic functions in $\mathbb{B}_{n}$. The system $\left\{p_{\nu}^{k}\right\}$ is orthonormal, but it is not complete in the space of continuous functions on $\partial \mathbb{B}_{n}$.

The explicit formula of the Cauchy-Szegö kernel

$$
H(z, \bar{\xi})=\frac{1}{\omega_{2 n-1}} \frac{1}{\left(1-z \bar{\xi}^{\prime}\right)^{n}}
$$

on $\partial \mathbb{B}_{n}$ was first deduced in Hua [7] by using the system $\left\{p_{v}^{k}\right\}$ and the relation

$$
H(z, \bar{\xi})=\sum_{k=0}^{\infty} \sum_{v=1}^{N_{k}} p_{v}^{k}(z) \overline{p_{v}^{k}(\xi)}, \quad z \in \mathbb{B}_{n}, \quad \xi \in \mathbb{B}_{n} .
$$

For $z, \omega \in \mathbb{B}_{n} \cup \partial \mathbb{B}_{n}$, the nonisotropic distance $d(z, \omega)$ is defined as

$$
d(z, \omega)=\left|1-z \bar{\omega}^{\prime}\right|^{1 / 2}
$$

It can be easily shown that $d(\cdot, \cdot)$ is a metric on $\partial \mathbb{B}_{n}$. For $\xi \in$ $\partial \mathbb{B}_{n}$ and $\varepsilon>0$, we define the ball corresponding to $d(\cdot, \cdot)$ as

$$
S(\xi, \varepsilon)=\left\{\eta \in \partial \mathbb{B}_{n}, d(\xi, \eta) \leq \varepsilon\right\} .
$$

The complement set of $S(\xi, \varepsilon)$ in $\partial \mathbb{B}_{n}$ is denoted by $S^{c}(\xi, \varepsilon)$. Set

$$
\begin{gathered}
\mathscr{A}=\{f: f \text { is holomorphic in } B(0,1+\delta) \\
\text { for some } \delta>0\} .
\end{gathered}
$$


If $f \in \mathscr{A}$, then

$$
f(z)=\sum_{k=0}^{\infty} \sum_{v=0}^{N_{k}} c_{k v} p_{v}^{k}(z),
$$

where $c_{k v}$ are the Fourier coefficients of $f$ :

$$
c_{k v}=\int_{\partial \mathbb{B}_{n}} \overline{p_{v}^{k}(\xi)} f(\xi) d \sigma(\xi),
$$

and for any positive integer $l$, the series

$$
\sum_{k=0}^{\infty} k^{l} \sum_{v=0}^{N_{k}} c_{k v} p_{v}^{k}(z)
$$

is uniformaly and absolutely convergent in any compact ball contained in $B(0,1+\delta)$ in which $f$ is defined.

Denote by $\mathcal{U}$ the unitary group of $\mathbb{C}^{n}$ consisting of all unitary operators on the Hilbert space $\mathbb{C}^{n}$ under the complex inner product $\langle z, w\rangle=z \bar{w}^{\prime}$. These are the linear operators $U$ that preserve inner products:

$$
\langle U z, U w\rangle=\langle z, w\rangle .
$$

Clearly, $\mathcal{U}$ is a compact subset of $O(2 n)$. It is easy to verify that $\mathscr{A}$ is invariant under $U \in \mathcal{U}$. If $f \in \mathscr{A}$, then $f$ is defined by its values on $\partial \mathbb{B}_{n}$. In Section 3, we treat $\left.f\right|_{\partial \mathbb{B}_{n}}$ as identical to $f \in \mathscr{A}$.

\section{The Kernel Generated by Holomorphic Multipliers}

Set

$$
\begin{gathered}
S_{\omega}=\{z \in \mathbb{C} z \neq 0 \text { and }|\arg z|<\omega\}, \\
S_{\omega}(\pi)=\{z \in \mathbb{C} z \neq 0,|\operatorname{Re}(z)| \leq \pi,|\arg ( \pm z)|<\omega\}, \\
W_{\omega}(\pi)=\{z \in \mathbb{C}|z \neq 0,| \operatorname{Re}(z) \mid \leq \pi, \operatorname{Im}(z)>0\} \\
\bigcup S_{\omega}(\pi), \\
H_{\omega}=\left\{z \in \mathbb{C} z=e^{i \omega}, \omega \in W_{\omega}(\pi)\right\} .
\end{gathered}
$$

The following function space is relevant.

Definition 2. Let $-1<s<\infty . H^{s}\left(S_{\omega}\right)$ is defined as the set of all holomorphic functions in $S_{\omega}$ such that

(a) $b$ is bounded for $|z| \leq 1$;

(b) $|b(z)| \leq C_{\mu}|z|^{s}, z \in S_{\mu}, 0<\mu<\omega$.

Remark 3. The classes $H^{s}\left(S_{\omega}\right)$ are generalizations of $H^{\infty}\left(S_{\omega}\right)$ which is introduced by McIntosh and his collaborators. We refer to Li et al. [9], McIntosh [10], McIntosh and Qian [2], Qian [11], and the reference therein for further information on $H^{\infty}\left(S_{\omega}\right)$.

Let

$$
\varphi_{b}(z)=\sum_{k=1}^{\infty} b(k) z^{k}
$$

Lemma 4. Let $b \in H^{s}\left(S_{\omega}\right),-1<s<\infty$. Then $\varphi_{b}$ can be holomorphically extended to $H_{\omega}$. Moreover, for $0<\mu<\mu^{\prime}<\omega$ and $l=0,1,2, \ldots$,

$$
\left|\left(z \frac{d}{d z}\right)^{l} \varphi_{b}(z)\right| \lesssim \frac{C_{\mu^{\prime}} l !}{\delta^{l}\left(\mu, \mu^{\prime}\right)|1-z|^{l+1+s}}, \quad z \in H_{\mu},
$$

where $\delta\left(\mu, \mu^{\prime}\right)=\min \left\{1 / 2, \tan \left(\mu, \mu^{\prime}\right\} ; C_{\mu^{\prime}}\right.$ are the constants in Definition 2.

Proof. Let

$$
\begin{gathered}
V_{\omega}=\{z \in \mathbb{C}: \operatorname{Im}(z)>0\} \bigcup S_{\omega} \bigcup\left(-S_{\omega}\right), \\
W_{\omega}=V_{\omega} \bigcap\{z \in \mathbb{C}:-\pi \leq \operatorname{Re} z \leq \pi\}
\end{gathered}
$$

and $\rho_{\theta}$ is the ray $r \exp (i \theta), 0<r<\infty$, where $\theta$ is chosen so that $\rho_{\theta} \subseteq S_{\omega}$. Define

$$
\Psi_{b}(z)=\frac{1}{2 \pi} \int_{\rho(\theta)} \exp (i \xi z) b(\xi) d \xi, \quad z \in V_{\omega},
$$

where $\exp (i z \xi)$ is exponentially decaying as $\xi \rightarrow \infty$ along $\rho_{\theta}$. Then we get

$$
\begin{aligned}
& \left.|| z\right|^{1+s} \Psi_{b}(z) \mid \\
& \quad=\left.\left|\frac{1}{2 \pi} \int_{\rho(\theta)} \exp (i \xi z)\right| z\right|^{1+s} b(\xi) d z \mid \\
& \quad \lesssim \frac{C_{\mu^{\prime}}}{2 \pi} \int_{0}^{\infty} \exp (-r|z| \sin (\theta+\arg z))(r|z|)^{s} d(r|z|)^{s} \\
& \quad \leq C_{\mu^{\prime}},
\end{aligned}
$$

which implies $\left|\Psi_{b}(z)\right| \lesssim 1 /|z|^{1+s}$. Define

$$
\begin{array}{r}
\psi_{b}(z)=2 \pi \sum_{n=-\infty}^{\infty} \Psi_{b}(z+2 n \pi), \\
z \in \bigcup_{n=-\infty}^{\infty}\left(2 n \pi+W_{\omega}\right) .
\end{array}
$$

It is easy to see that $\psi_{b}$ is holomorphically and $2 \pi$-periodically defined in the described region, and $\left|\psi_{b}(z)\right| \lesssim 1 /|z|^{1+s}$. Let

$$
\varphi_{b}(z)=\psi_{b}\left(\frac{\log z}{i}\right) \text {. }
$$

For $z \in \exp \left(i S_{\omega}\right)$, we write $z=e^{i u}$, where $u \in S_{\omega}$. Then $\sin (|u| / 2) \lesssim|u| / 2$. This implies that $2-2 \cos |u| \lesssim|u|^{2}$ and $\left|1-e^{i|u|}\right| \lesssim|u|$. Therefore, (29) gives

$$
\left|\varphi_{b}(z)\right| \lesssim \frac{C_{\mu^{\prime}}}{|\log z|^{1+s}} \lesssim \frac{C_{\mu^{\prime}}}{\left.|\log | z\right|^{1+s}} \lesssim \frac{C_{\mu^{\prime}}}{|1-z|^{1+s}} .
$$

Take the ball

$$
B(z, r)=\left\{\xi:|z-\xi|<\delta\left(\mu, \mu^{\prime}\right)|1-z|\right\} .
$$


Applying Cauchy's integral formula, we obtain

$$
\varphi_{b}^{(l)}(z)=\frac{l !}{2 \pi i} \int_{\partial B(z, r)} \frac{\varphi(\eta)}{(\eta-z)^{1+l}} d \eta .
$$

For any $\eta \in \partial B(z, r)$, we have $|\eta-z| \geq\left(1-\delta\left(\mu, \mu^{\prime}\right)\right)|1-z|$. Then we have

$$
\begin{aligned}
\left|\varphi_{b}^{(l)}(z)\right| & \lesssim \frac{l !\|b\|_{H^{s}\left(S_{\omega}^{c}\right)}}{\delta^{l}\left(\mu, \mu^{\prime}\right)|1-z|^{l}}\left|\int_{\partial B(z, r)} \frac{1}{|1-\eta|^{1+s}} d \eta\right| \\
& \lesssim \frac{l !}{\delta^{l}\left(\mu, \mu^{\prime}\right)|1-z|^{l+1+s}}
\end{aligned}
$$

Theorem 5. Let $b \in H^{s}\left(S_{\omega}\right)$ and

$$
\begin{array}{r}
H_{b}(z, \bar{\xi})=\sum_{k=1}^{\infty} b(k) \sum_{v=1}^{N_{k}} p_{v}^{k}(z) \overline{p_{v}^{k}(\xi)} \\
z \in \mathbb{B}_{n}, \quad \xi \in \partial \mathbb{B}_{n} .
\end{array}
$$

Then

$$
H_{b}(z, \bar{\xi})=\left.\frac{1}{(n-1) ! \omega_{2 n-1}}\left(r^{n-1} \varphi_{b}(r)\right)^{(n-1)}\right|_{r=z \bar{\xi}^{\prime}}
$$

is holomorphically defined for $z \in \mathbb{B}_{n}, \xi \in \partial \mathbb{B}_{n}$ such that $z \bar{\xi}^{\prime} \in$ $H_{\omega}$, where $\varphi_{b}$ is the function defined in Lemma 4, Moreover, for $0<\mu<\mu^{\prime}<\omega$ and $l=0,1,2, \ldots$,

$$
\left|D_{z}^{l} H_{b}(z, \bar{\xi})\right| \lesssim \frac{C_{\mu^{\prime}} l !}{\delta^{l}\left(\mu, \mu^{\prime}\right)\left|1-z \bar{\xi}^{\prime}\right|^{n+l+s}}, \quad z \bar{\xi}^{\prime} \in H_{\mu}
$$

where $\delta\left(\mu, \mu^{\prime}\right)=\min \left\{1 / 2, \tan \left(\mu^{\prime}-\mu\right)\right\}, C_{\mu^{\prime}}$ are the constant in the definition of the function space $H^{s}\left(S_{\omega}\right)$.

Proof. Recall that

$$
\begin{aligned}
\varphi_{b}(z) & =\sum_{k=1}^{\infty} b(k) z^{k} ; \\
r^{n-1} \varphi_{b}(r) & =\sum_{k=1}^{\infty} b(k) r^{n+k-1} .
\end{aligned}
$$

Then we have

$$
\begin{aligned}
& \frac{1}{(n-1) !}\left(r^{n-1} \varphi_{b}(r)\right)^{(n-1)} \\
& =\frac{1}{(n-1) !} \sum_{k=1}^{\infty} b(k)(n+k-1)(n+k-2) \cdots(k+1) r^{k} \\
& =\sum_{k=1}^{\infty} b(k) r^{k} \frac{(n+k-1) !}{(n-1) ! k !} \\
& =\sum_{k=1}^{\infty} \frac{(n+k-1)(n+k-2)(n+1) n}{k !} b(k) r^{k} .
\end{aligned}
$$

Therefore,

$$
\begin{aligned}
& \left.\frac{1}{(n-1) !}\left(r^{n-1} \varphi_{b}(r)\right)^{(n-1)}\right|_{r=z \bar{\xi}^{\prime}} \\
& =\sum_{k=1}^{\infty} b(k) \frac{(n+k-1)(n+k-2)(n+1) n}{k !}\left(z \bar{\xi}^{\prime}\right)^{k} \\
& =\omega_{2 n-1} \sum_{k=1}^{\infty} b(k) \sum_{v=1}^{N_{k}} p_{v}^{k}(z) \overline{p_{v}^{k}(\xi)} \\
& =\omega_{2 n-1} H_{b}(z, \bar{\xi}) .
\end{aligned}
$$

By [4, Theorem 3], we could obtain the following result.

Theorem 6. Let $s$ be a negative integer. If $b \in H^{s}\left(S_{\omega, \pm}\right)$,

$$
\begin{array}{r}
H_{b}(z, \xi)=\sum_{k=1}^{\infty} b(k) \sum_{v=1}^{N_{k}} p_{v}^{k}(z) p_{\mu}^{l}(\xi), \\
z \in \mathbb{B}, \quad \xi \in \partial \mathbb{B}_{n},
\end{array}
$$

then

$$
\left|D_{z}^{l} H_{b}(z, \bar{\xi})\right| \lesssim \frac{C_{\mu} l !\left[|\ln | 1-z \bar{\xi}^{\prime}||+1\right]}{\delta^{l}\left(\mu, \mu^{\prime}\right)\left|1-z \bar{\xi}^{\prime}\right|^{n+l+s}} .
$$

Proof. The proof is similar to Theorem 5. we omit it.

\section{Sobolev Spaces and Unbounded Fourier Multipliers}

4.1. Integral Representation of Multipliers. Given $b \in H^{s}\left(S_{\omega}\right)$, we define the Fourier multiplier operator $M_{b}: \mathscr{A} \rightarrow \mathscr{A}$ by

$$
M_{b}(f)(\xi)=\sum_{k=1}^{\infty} b(k) \sum_{v=0}^{N_{k}} c_{k v} p_{v}^{k}(\xi), \quad \xi \in \partial \mathbb{B}_{n}
$$

where $\left\{c_{k v}\right\}$ are the Fourier coefficients of the test function $f \in$ $\mathscr{A}$.

For the above operator $M_{b}$, the Plemelj type formula holds.

Theorem 7. Let $b \in H^{s}\left(S_{\omega}\right), s>0$. Take $b_{1}(z)=z^{-s_{1}} b(z)$, where $s_{1}=[s]+1$. Operator $M_{b}$ has a singular integral expression. For $f \in \mathscr{A}$,

$$
\begin{aligned}
M_{b}(f)(\xi)=\lim _{\varepsilon \rightarrow 0}\left[\int_{S^{c}(\xi, \varepsilon)} H_{b_{1}}(\xi, \bar{\eta}) D_{\eta}^{s_{1}} f(\eta) d \sigma(\eta)\right. \\
\\
\left.\quad+\left(D_{z}^{s_{1}} f\right)(\xi) \int_{S^{c}(\xi, \varepsilon)} H_{b_{1}}(\xi, \bar{\eta}) d \sigma(\eta)\right],
\end{aligned}
$$

where $\int_{S(\xi, \varepsilon)} H_{b_{1}}(\xi, \bar{\eta}) d \sigma(\eta)$ is a bounded function of $\xi \in \partial \mathbb{B}_{n}$ and $\varepsilon$. 
Proof. Let

$$
M_{b}(f)(\rho \xi)=\sum_{k=1}^{\infty} b(k) \sum_{v=1}^{N_{k}} c_{k v} p_{v}^{k}(\rho \xi), \quad \xi \in \partial \mathbb{B}_{n},
$$

where

$$
c_{k v}=\int_{\partial B} \overline{p_{v}^{k}(\eta)} f(\eta) d \sigma(\eta) .
$$

We can see that

$$
\begin{aligned}
D_{z} z^{[l]} \\
\quad=\sqrt{\frac{l !}{l_{1} ! l_{2} ! \cdots l_{n} !} \sum_{k=1}^{n} z_{k} \frac{\partial}{\partial z_{k}}\left(z_{1}^{l_{1}} z_{2}^{l_{2}} \cdots z_{n}^{l_{n}}\right)} \\
=\sqrt{\frac{l !}{l_{1} ! l_{2} ! \cdots l_{n} !} \sum_{k=1}^{n} z_{k} l_{k} z_{1}^{l_{1}} z_{2}^{l_{2}} \cdots z_{k-1}^{l_{k-1}} z_{k}^{l_{k}-1} z_{k+1}^{l_{k+1}} \cdots z_{n}^{l_{n}}} \\
=\sqrt{\frac{l !}{l_{1} ! l_{2} ! \cdots l_{n} !}}\left(\sum_{k=1}^{n} l_{k}\right) z_{1}^{l_{1}} z_{2}^{l_{2}} \cdots z_{n}^{l_{n}} \\
=l z^{[l]},
\end{aligned}
$$

which implies that $D_{z} p_{v}^{k}=k p_{v}^{k}$. Then, we have

$$
\begin{aligned}
M_{b} & (f)(\rho \xi) \\
= & \sum_{k=1}^{\infty} b(k) \sum_{v=1}^{N_{k}} \int_{\partial B} p_{v}^{k}(\rho \xi) \overline{p_{v}^{k}(\eta)} f(\eta) d \sigma(\eta) \\
= & \sum_{k=1}^{\infty} b(k) \frac{1}{k^{s_{1}}} \sum_{v=1}^{N_{k}} \int_{\partial B} p_{v}^{k}(\rho \xi) k^{s_{1}} \overline{p_{v}^{k}(\eta)} f(\eta) d \sigma(\eta) \\
= & \sum_{k=1}^{\infty} b(k) \frac{1}{k^{s_{1}}} \sum_{v=1}^{N_{k}} \int_{\partial B} p_{v}^{k}(\rho \xi) D_{\eta}^{s_{1}} \overline{p_{v}^{k}(\eta)} f(\eta) d \sigma(\eta) .
\end{aligned}
$$

By integration by parts,

$$
\begin{aligned}
M_{b} & (f)(\rho \xi) \\
= & \sum_{k=1}^{\infty} b(k) \frac{1}{k^{s_{1}}} \sum_{v=1}^{N_{k}} \int_{\partial B} p_{v}^{k}(\rho \xi) \overline{p_{v}^{k}(\eta)}\left(D_{\eta}^{s_{1}} f\right)(\eta) d \sigma(\eta) \\
= & \sum_{k=1}^{\infty} b_{1}(k) \sum_{v=1}^{N_{k}} \int_{\partial B} p_{v}^{k}(\rho \xi) \overline{p_{v}^{k}(\eta)}\left(D_{\eta}^{s_{1}} f\right)(\eta) d \sigma(\eta) .
\end{aligned}
$$

For any $\varepsilon>0$, we have

$$
\begin{aligned}
& M_{b}(f)(\rho \xi) \\
& \quad=\int_{S^{c}(\xi, \varepsilon)} H_{b_{1}}(\rho \xi, \bar{\eta}) D_{\eta}^{s_{1}} f(\eta) d \sigma(\eta) \\
& \quad+\int_{S(\xi, \varepsilon)} H_{b_{1}}(\rho \xi, \bar{\eta})\left(-D_{\xi}^{s_{1}} f(\xi)+D_{\eta}^{s_{1}} f(\eta)\right) d \sigma(\eta)
\end{aligned}
$$

$$
\begin{aligned}
& +D_{\xi}^{s_{1}} f(\xi) \int_{S(\xi, \varepsilon)} H_{b_{1}}(\rho \xi, \bar{\eta}) d \sigma(\eta) \\
= & I_{1}(\rho, \varepsilon)+I_{2}(\rho, \varepsilon)+D_{\xi}^{s_{1}} f(\xi) I_{3}(\rho, \varepsilon),
\end{aligned}
$$

where

$$
\begin{gathered}
I_{1}(\rho, \varepsilon)=\int_{S^{c}(\xi, \varepsilon)} H_{b_{1}}(\rho \xi, \bar{\eta}) D_{\eta}^{s_{1}} f(\eta) d \sigma(\eta), \\
I_{2}(\rho, \varepsilon)=\int_{S(\xi, \varepsilon)} H_{b_{1}}(\rho \xi, \bar{\eta})\left(-D_{\xi}^{s_{1}} f(\xi)+D_{\eta}^{s_{1}} f(\eta)\right) d \sigma(\eta), \\
I_{3}(\rho, \varepsilon)=\int_{S(\xi, \varepsilon)} H_{b_{1}}(\rho \xi, \bar{\eta}) d \sigma(\eta) .
\end{gathered}
$$

$$
\begin{aligned}
& \text { For } \rho \rightarrow 1-0 \text {, we have } \\
& \begin{aligned}
\lim _{\rho \rightarrow 1-0} I_{1}(\rho, \varepsilon) & =\lim _{\rho \rightarrow 1-0} \int_{S^{c}(\xi, \varepsilon)} H_{b_{1}}(\rho \xi, \bar{\eta}) D_{\eta}^{s_{1}} f(\eta) d \sigma(\eta) \\
& =\int_{S^{c}(\xi, \varepsilon)} H_{b_{1}}(\xi, \bar{\eta}) D_{\eta}^{s_{1}} f(\eta) d \sigma(\eta) .
\end{aligned}
\end{aligned}
$$

Now we consider $I_{2}(\rho, \varepsilon)$. Let $\xi=[1,0, \ldots, 0]$. For $\eta \in \partial \mathbb{B}_{n}$, write

$$
\begin{aligned}
\eta_{1}=r e^{i \theta}, \quad \eta_{2} & =v_{2}, \quad \eta_{3}=v_{3}, \ldots, \quad \eta_{n}=v_{n} ; \\
v & =\left[v_{2}, v_{3}, \ldots, v_{n}\right] .
\end{aligned}
$$

For such $\eta \in \partial \mathbb{B}_{n}, v \bar{v}^{\prime}=1-r^{2}$. Without loss of generality, assume $\xi=1$. We get

$$
\begin{aligned}
\left|1-\xi \bar{\eta}^{\prime}\right|^{1 / 2} & =\left|1-r e^{i \theta}\right|^{1 / 2} \\
& =\left[(1-r \cos \theta)^{2}+(r \sin \theta)^{2}\right]^{1 / 4} \leq \varepsilon,
\end{aligned}
$$

which implies that

$$
\cos \theta \geq \frac{1+r^{2}-\varepsilon^{4}}{2 r} .
$$

The above estimate implies

$$
S(\xi, \varepsilon)=\left\{\eta \mid v \bar{v}^{\prime}=1-r^{2}, \cos \theta \geq \frac{1+r^{2}-\varepsilon^{4}}{2 r}\right\} .
$$

Since

$$
\frac{1+r^{2}-\varepsilon^{4}}{2 r} \leq \cos \theta \leq 1,
$$

we obtain $1-r \leq \varepsilon^{2}$ and then

$$
v \bar{v}^{\prime}=1-r^{2} \leq 1-\left(1-\varepsilon^{2}\right)^{2}=2 \varepsilon^{2}-\varepsilon^{4} .
$$

Denote

$$
a=a(r, \varepsilon)=\arccos \left(\frac{1+r^{2}-\varepsilon^{4}}{2 r}\right) .
$$

Since $(1-r)^{2} \leq \varepsilon^{4}$ and $1-y=O\left(\arccos ^{2} y\right)$, we get $a=O\left(\varepsilon^{2}\right)$. It is easy to see

$$
\begin{aligned}
|\xi-\eta|^{2} & =\left|1-r e^{i \theta}\right|^{2}+\sum_{k=2}^{n}\left|v_{k}\right|^{2} \\
& =\left(1+r^{2}-2 r \cos \theta\right)+\left(1-r^{2}\right)
\end{aligned}
$$




$$
\begin{aligned}
& =2-2 r \cos \theta, \\
d^{4}(\xi, \eta) & =1+r^{2}-2 r \cos \theta \\
& =(2-2 r \cos \theta)-\left(1-r^{2}\right) \\
& =|\xi-\eta|^{2}-(1+r)(1-r),
\end{aligned}
$$

that is, $d^{2}(\xi, \eta) \leq|\xi-\eta|$. Because

$$
d^{2}(\xi, \eta)=\left[1+r^{2}-2 r \cos \theta\right]^{1 / 2} \geq 1-r,
$$

then we have $1-r \leq d^{2}(\xi, \eta)$, so

$$
|\xi-\eta|^{2} \leq d^{4}(\xi, \eta)+(1+r) d^{2}(\xi, \eta) .
$$

Since $d^{2}(\xi, \eta) \leq 2$, then

$$
|\xi-\eta|^{2} \leq 2 d^{2}(\xi, \eta)+2 d^{2}(\xi, \eta)=4 d^{2}(\xi, \eta),
$$

that is

$$
|\xi-\eta| \leq 2 d(\xi, \eta) .
$$

Since $f \in \mathscr{A}$, we have

$$
|f(\xi)-f(\eta)| \leqslant|\xi-\eta| \leqslant d(\xi, \eta) .
$$

For $\rho \in(0,1)$,

$$
\begin{aligned}
\left|I_{2}(\rho, \varepsilon)\right| & \lesssim \int_{S(\xi, \varepsilon)}\left|H_{b_{1}}(\rho \xi, \bar{\eta})\right||f(\xi)-f(\eta)| d \sigma(\eta) \\
& \lesssim \int_{S(\xi, \varepsilon)} \frac{d(\xi, \eta)}{\left|1-\xi \bar{\eta}^{\prime}\right|^{n}} d \sigma(\eta) \\
& \lesssim \int_{\bar{v}^{\prime} \leq 2 \varepsilon^{2}-\varepsilon^{4}} \int_{-a}^{a} \frac{1}{\left|1-r e^{i \theta}\right|^{n-1 / 2}} d \theta d v .
\end{aligned}
$$

For $n=2$,

$$
\begin{aligned}
\frac{1}{2 a} \int_{-a}^{a} \frac{1}{\left|1-r e^{i \theta}\right|^{2-1 / 2}} d \theta & \leq\left(\frac{1}{2 a} \int_{-a}^{a} \frac{1}{\left|1-r e^{i \theta}\right|^{2}} d \theta\right)^{3 / 4} \\
& \leq\left(\frac{1}{2 a} \int_{-\pi}^{\pi} \frac{1}{\left|1-r e^{i \theta}\right|^{2}} d \theta\right)^{3 / 4} \\
& \leq\left(\frac{1}{2 a}\right)^{3 / 4} \frac{1}{\left(1-r^{2}\right)^{3 / 4}} .
\end{aligned}
$$

Then we get

$$
\begin{aligned}
\left|I_{2}(\rho, \varepsilon)\right| & \leqslant \int_{\nu \bar{v}^{\prime} \leq 2 \varepsilon^{2}-\varepsilon^{4}} a^{1 / 4} \frac{1}{\left(1-r^{2}\right)^{3 / 4}} d v \\
& \leqslant \varepsilon^{1 / 2} \int_{v \bar{v}^{\prime} \leq 2 \varepsilon^{2}-\varepsilon^{4}} \frac{1}{\left(v \bar{v}^{\prime}\right)^{3 / 4}} d v
\end{aligned}
$$

$$
\begin{aligned}
& =\varepsilon^{1 / 2} \int_{0}^{\sqrt{2 \varepsilon^{2}-\varepsilon^{4}}} \frac{t}{t^{3 / 2}} d t \\
& \leq \varepsilon \longrightarrow 0 .
\end{aligned}
$$

For $n>2$, we have

$$
\begin{aligned}
\int_{-a}^{a} \frac{1}{\left|1-r e^{i \theta}\right|^{n-1 / 2}} d \theta & \lesssim \int_{-a}^{a} \frac{\left|1-r^{2}\right|^{n-1 / 2-2}}{\left|1-r e^{i \theta}\right|^{n-1 / 2}} \frac{1}{\left|1-r^{2}\right|^{n-1 / 2-2}} d \theta \\
& \lesssim \frac{1}{\left|1-r^{2}\right|^{n-1 / 2-1}} \int_{-\pi}^{\pi} \frac{1}{\left|1-r e^{i \theta}\right|^{2}} d \theta \\
& \lesssim \frac{1}{\left|1-r^{2}\right|^{n-1 / 2-1}} .
\end{aligned}
$$

Then, we get

$$
\left|I_{2}(\rho, \varepsilon)\right| \lesssim \int_{0}^{\sqrt{2 \varepsilon^{2}-\varepsilon^{4}}} t^{2 n-3} \frac{1}{t^{2 n-3}} d t \lesssim \sqrt{2 \varepsilon^{2}} \longrightarrow 0 .
$$

Now we prove if $\rho \rightarrow 1-0, I_{3}(\rho, \varepsilon)$ has a limit uniformly bounded for $\varepsilon$ near 0 . Integrating as before, we have

$$
\begin{aligned}
I_{3}(\rho, \varepsilon) & =\int_{S(\xi, \varepsilon)} H_{b_{1}}(\rho \xi, \bar{\eta}) d \sigma(\eta) \\
& =\left.\int_{\nu \bar{v}^{\prime} \leq 2 \varepsilon^{2}-\varepsilon^{4}} \int_{-a}^{a}\left(t^{n-1} \varphi_{b_{1}}(t)\right)^{(n-1)}\right|_{t=\rho r e^{i \theta}} d \theta d v .
\end{aligned}
$$

Let $s=\rho r e^{i \theta}$. Then $d s=i s d \theta$. We get

$$
I_{3}(\rho, \varepsilon)=-i \int_{v \bar{v}^{\prime} \leq 2 \varepsilon^{2}-\varepsilon^{4}} \int_{\rho r e^{-i a}}^{\rho r e^{i a}}\left(s^{n-1} \varphi_{b_{1}}(s)\right)^{(n-1)} d s d v .
$$

By integration by parts, the inside integral with respect to the variable $t$ becomes

$$
\begin{aligned}
\int_{-a}^{a} & \left.\left(t^{n-1} \varphi_{b_{1}}(t)\right)^{(n-1)}\right|_{t=\rho r e^{i \theta}} d \theta \\
= & {\left.\left[\sum_{k=1}^{n-1}(k-1) ! \frac{\left(t^{n-1} \varphi_{b_{1}}(t)\right)^{(n-k-1)}}{t^{k}}\right]\right|_{\rho r e^{-i a}} ^{\rho r e^{i a}} } \\
& +(n-1) ! \int_{\rho r e^{-i a}}^{\rho r e^{i a}} \frac{\varphi_{b_{1}}(t)}{t} d t \\
= & \sum_{k=1}^{n-1}\left[J_{k}(t)\right]_{\rho r e^{-i a}}^{\rho r i a}+L(r, a) .
\end{aligned}
$$

We first estimate $J_{k}$,

$$
\begin{aligned}
& \int_{v \bar{v}^{\prime} \leq 2 \varepsilon^{2}-\varepsilon^{4}} J_{k}\left(\rho r e^{ \pm i a}\right) d v \\
& \quad \leq \int_{v \bar{v}^{\prime} \leq 2 \varepsilon^{2}-\varepsilon^{4}}(k-1) ! \frac{\left(\rho r e^{ \pm i a}\right)^{k}}{\left(\rho r e^{ \pm i a}\right)^{k}} \frac{1}{\left|1-\rho r e^{ \pm i a}\right|^{n-k}} d v \\
& \quad \leq \int_{\nu \bar{v}^{\prime} \leq 2 \varepsilon^{2}-\varepsilon^{4}} \frac{1}{\left|1-\rho r e^{ \pm i a}\right|^{n-k}} d v .
\end{aligned}
$$




$$
\begin{aligned}
& \text { Because }\left|1-\rho r e^{ \pm i a}\right|^{2}=1+\rho^{2} r^{2}-2 \rho r \cos a, \\
& \qquad \begin{array}{c}
\left|1-\rho r e^{ \pm i a}\right|^{2}-\left|1-r e^{ \pm i a}\right|^{2} \\
=\rho^{2} r^{2}-2 \rho r \cos a-\left(r^{2}-2 r \cos a\right) \\
=r^{2}\left(\rho^{2}-1\right)+2 r \cos a(1-\rho) .
\end{array}
\end{aligned}
$$

Since $\cos a=\left(1+r^{2}-\varepsilon^{4}\right) / 2 r$, we have

$$
\begin{aligned}
\mid 1 & -\left.\rho r e^{ \pm i a}\right|^{2}-\left|1-r e^{ \pm i a}\right|^{2} \\
& =r^{2}\left(\rho^{2}-1\right)+\left(1+r^{2}-\varepsilon^{4}\right)(1-\rho) \\
& =(1-\rho)\left[1+r^{2}-\varepsilon^{4}-(1+\rho) r^{2}\right] \\
& =(1-\rho)\left(1-\rho r^{2}-\varepsilon^{4}\right)>0 .
\end{aligned}
$$

So

$$
\left|1-\rho r e^{ \pm i a}\right| \geq\left|1-r e^{ \pm i a}\right|=\varepsilon^{2} .
$$

For $k$, when $\varepsilon \rightarrow 0$, we obtain

$$
\begin{aligned}
\int_{v \vec{v}^{\prime} \leq 2 \varepsilon^{2}-\varepsilon^{4}} J_{k}\left(\rho r e^{ \pm i a}\right) d v & \lesssim \frac{1}{\varepsilon^{2 n-2 k}} \int_{v \bar{v}^{\prime} \leq 2 \varepsilon^{2}-\varepsilon^{4}} d v \\
& \lesssim \frac{1}{\varepsilon^{2 n-2 k}} \int_{0}^{\sqrt{2 \varepsilon^{2}-\varepsilon^{4}}} t^{2 n-3} d t \\
& \lesssim \frac{\varepsilon^{2 n-2}}{\varepsilon^{2 n-2 k}} \lesssim 1 .
\end{aligned}
$$

On the other hand,

$$
\begin{aligned}
& (n-1) ! \int_{\rho r e^{-i a}}^{\rho r e^{i a}} \frac{\varphi_{b_{1}}(t)}{t} d t \\
& \quad=\left.i(n-1) ! \int_{-a}^{a} \varphi_{b_{1}}(t)\right|_{t=\rho r e^{i \theta}} d \theta \\
& \quad \lesssim 1, \quad(\text { when } \rho \longrightarrow 0)
\end{aligned}
$$

that implies

$$
\int_{v \bar{v}^{\prime} \leq 2 \varepsilon^{2}-\varepsilon^{4}} L(\rho r, a) d v .
$$

4.2. Sobolev Spaces on $\partial \mathbb{B}_{n}$ via Fourier Multipliers. Sobolev spaces on the $n$-complex unit sphere $\partial \mathbb{B}_{n}$ are defined as follows. We define the fractional integral operator $\mathscr{I}^{s}$ on $\partial \mathbb{B}_{n}$ as follows. Let

$$
f(z)=\sum_{k=0}^{\infty} \sum_{v=0}^{N_{k}} c_{k v} p_{v}^{k}(z) .
$$

For $-\infty<s<\infty$, the operator $\mathscr{I}^{s}$ is defined by

$$
\mathscr{I}^{s} f(z)=\sum_{k=0}^{\infty} \sum_{v=0}^{N_{k}} k^{s} c_{k v} p_{v}^{k}(z) \text {. }
$$

For $s \in \mathbb{Z}_{+}$, we can see that the operators $\mathscr{I}^{s}$ become the ordinary differential operators with higher orders.
Theorem 8. Let $s \in \mathbb{Z}_{+}$. $D_{z}^{s}=\mathscr{I}^{s}$ on $L^{2}\left(\partial \mathbb{B}_{n}\right)$.

Proof. Without loss of generality, we assume that $f \in \mathscr{A}$. Then

$$
f(z)=\sum_{k=0}^{\infty} \sum_{v=0}^{N_{k}} c_{k v} p_{v}^{k}(z),
$$

where $c_{k v}$ are the Fourier coefficients of $f$ :

$$
c_{k v}=\int_{\partial \mathbb{B}_{n}} \overline{p_{v}^{k}(\xi)} f(\xi) d \sigma(\xi) .
$$

So

$$
\begin{aligned}
D_{z}^{s} f(z) & =\sum_{k=0}^{\infty} \sum_{v=0}^{N_{k}} \int_{\partial \mathbb{B}_{n}} \overline{p_{v}^{k}(\xi)} f(\xi) d \sigma(\xi) D_{z}^{s}\left(p_{v}^{k}\right)(z) \\
& =\sum_{k=0}^{\infty} k^{s} \sum_{v=0}^{N_{k}} \int_{\partial \mathbb{B}_{n}} \overline{p_{v}^{k}(\xi)} f(\xi) d \sigma(\xi) p_{v}^{k}(z) .
\end{aligned}
$$

Definition 9. Let $s \in[0,+\infty)$. The Sobolev norm $\|\cdot\|_{W^{2, s}\left(\partial \mathbb{B}_{n}\right)}$ on $\partial \mathbb{B}_{n}$ is defined as

$$
\|f\|_{W^{2, s}\left(\partial \mathbb{B}_{n}\right)}=:\left\|\mathscr{J}^{s} f\right\|_{2}<\infty .
$$

The Sobolev spaces on $\partial \mathbb{B}_{n}$ are defined as the closure of $\mathscr{A}$ under the norm $\|\cdot\|_{W^{2, s}\left(\partial \mathbb{B}_{n}\right)}$, that is, $W^{2, s}\left(\partial \mathbb{B}_{n}\right)=\overline{\mathscr{A}}^{\|\cdot\|_{W^{2, s}\left(\partial \mathbb{B}_{n}\right)}}$.

Remark 10. By the Plancherel theorem, $f \in W^{2, s}\left(\partial \mathbb{B}_{n}\right)$ if and only if

$$
\left(\sum_{k=1}^{\infty} k^{2 s} \sum_{v=0}^{N_{k}}\left|c_{k v}\right|^{2}\right)^{1 / 2}<\infty .
$$

Now we consider the Sobolev boundedness of $M_{b}$.

Theorem 11. Given $r, s \in[0,+\infty)$ and $b \in H^{s}\left(S_{\omega}\right)$. The Fourier multiplier operator $M_{b}$ is bounded from $W^{2, r+s}\left(\partial \mathbb{B}_{n}\right)$ to $W^{2, r}\left(\partial \mathbb{B}_{n}\right)$.

Proof. Write

$$
\mathscr{I}^{s} f(z)=\sum_{k=0}^{\infty} \sum_{v=0}^{N_{k}} c_{k v}^{s} p_{v}^{k}(z) .
$$

By the orthogonality of $\left\{p_{v}^{k}\right\}$, we can see that $c_{k v}^{s}=k^{s} c_{k v}$. Let $b(z)=z^{-s} b(z)$. Because $b \in H^{s}\left(S_{\omega}\right)$, we can see that $b_{1} \in$ $H^{\infty}\left(S_{\omega}\right)$. This implies that

$$
\begin{aligned}
\mathscr{J}^{r}\left(M_{b}(f)\right)(\xi) & =\sum_{k=1}^{\infty} b(k) k^{r} \sum_{v=0}^{N_{k}} c_{k v} p_{v}^{k}(\xi) \\
& =\sum_{k=1}^{\infty} b_{1}(k) k^{r+s} \sum_{v=0}^{N_{k}} c_{k v} p_{v}^{k}(\xi) \\
& =M_{b_{1}}\left(\mathscr{J}^{r+s} f\right)(\xi) .
\end{aligned}
$$


Finally, by [5, Theorem 3], we can see that

$$
\begin{aligned}
\left\|M_{b}(f)\right\|_{W^{2, r}} & =\left\|\mathscr{J}^{r}\left(M_{b}(f)\right)\right\|_{2} \\
& =\left\|M_{b_{1}}\left(\mathscr{I}^{r+s} f\right)\right\|_{2} \\
& \leq C\left\|\mathscr{I}^{r+s} f\right\|_{2} .
\end{aligned}
$$

This completes the proof of Theorem 11.

\section{Conflict of Interests}

The authors declare that they have no conflict of interests in this submitted paper.

\section{Acknowledgments}

This project is supported by NSFC Grant no. 11171203; New Teacher's Fund for Doctor Stations, Ministry of Education, Grant no. 20114402120003; Guangdong Natural Science Foundation S2011040004131; Foundation for Distinguished Young Talents in Higher Education of Guangdong, China, LYM11063. Tao Qian is supported by MYRG116(Y1-L3)-FST13-QT; MYRG115(Y1-L4)-FST13-QT; FDCT 098/2012/A3.

\section{References}

[1] G. Gaudry, T. Qian, and S. Wang, "Boundedness of singular integral operators with holomorphic kernels on star-shaped closed Lipschitz curves," Colloquium Mathematicum, vol. 70, no. 1, pp. 133-150, 1996.

[2] A. McIntosh and T. Qian, "Convolution singular integral operators on Lipschitz curves," in Harmonic Analysis, vol. 1494 of Lecture Notes in Mathematics, pp. 142-162, Springer, Berlin, Germany, 1991.

[3] T. Qian, "Singular integrals with holomorphic kernels and $H_{\infty}$ Fourier multipliers on star-shaped closed Lipschitz curves," Studia Mathematica, vol. 123, no. 3, pp. 195-216, 1997.

[4] T. Qian, "A holomorphic extension result," Complex Variables. Theory and Application, vol. 32, no. 1, pp. 59-77, 1997.

[5] M. Cowling and T. Qian, "A class of singular integrals on the $n$ complex unit sphere," Science in China. Series A, vol. 42, no. 12, pp. 1233-1245, 1999.

[6] S. Gong, Integrals of Cauchy Type on the Ball, vol. 1 of Monographs in Analysis, International Press, Cambridge, Mass, USA, 1993.

[7] L. K. Hua, Harmonic Analysis of Functions of Several Complex Variables in the Classical Domains, American Mathematical Society, Providence, RI, USA, 1963.

[8] W. Rudin, Function theory in the unit ball of $\mathbb{C}^{n}$, Grundlehren der Mathematischen Wissenschaften, Springer, Berlin, Germany, 2008.

[9] C. Li, A. McIntosh, and S. Semmes, "Convolution singular integrals on Lipschitz surfaces," Journal of the American Mathematical Society, vol. 5, no. 3, pp. 455-481, 1992.

[10] A. McIntosh, "Operators which have an $H_{\infty}$ functional calculus," in Proceedings of the Mini Conference on Operator Theory and Partial Differential Equations, Center for Mathematical Analysis, vol. 14, ANU, Canberra, Australia.
[11] T. Qian, "Fourier analysis on starlike Lipschitz surfaces," Journal of Functional Analysis, vol. 183, no. 2, pp. 370-412, 2001. 


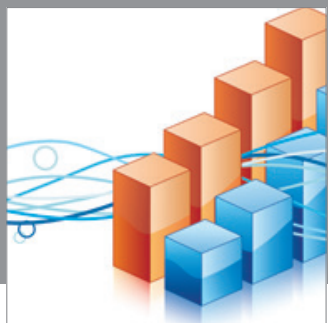

Advances in

Operations Research

mansans

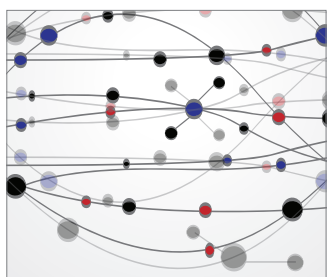

The Scientific World Journal
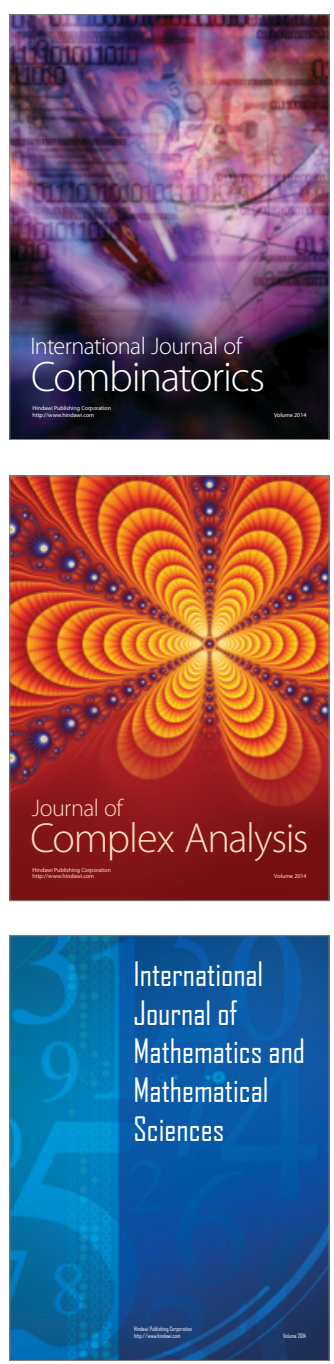
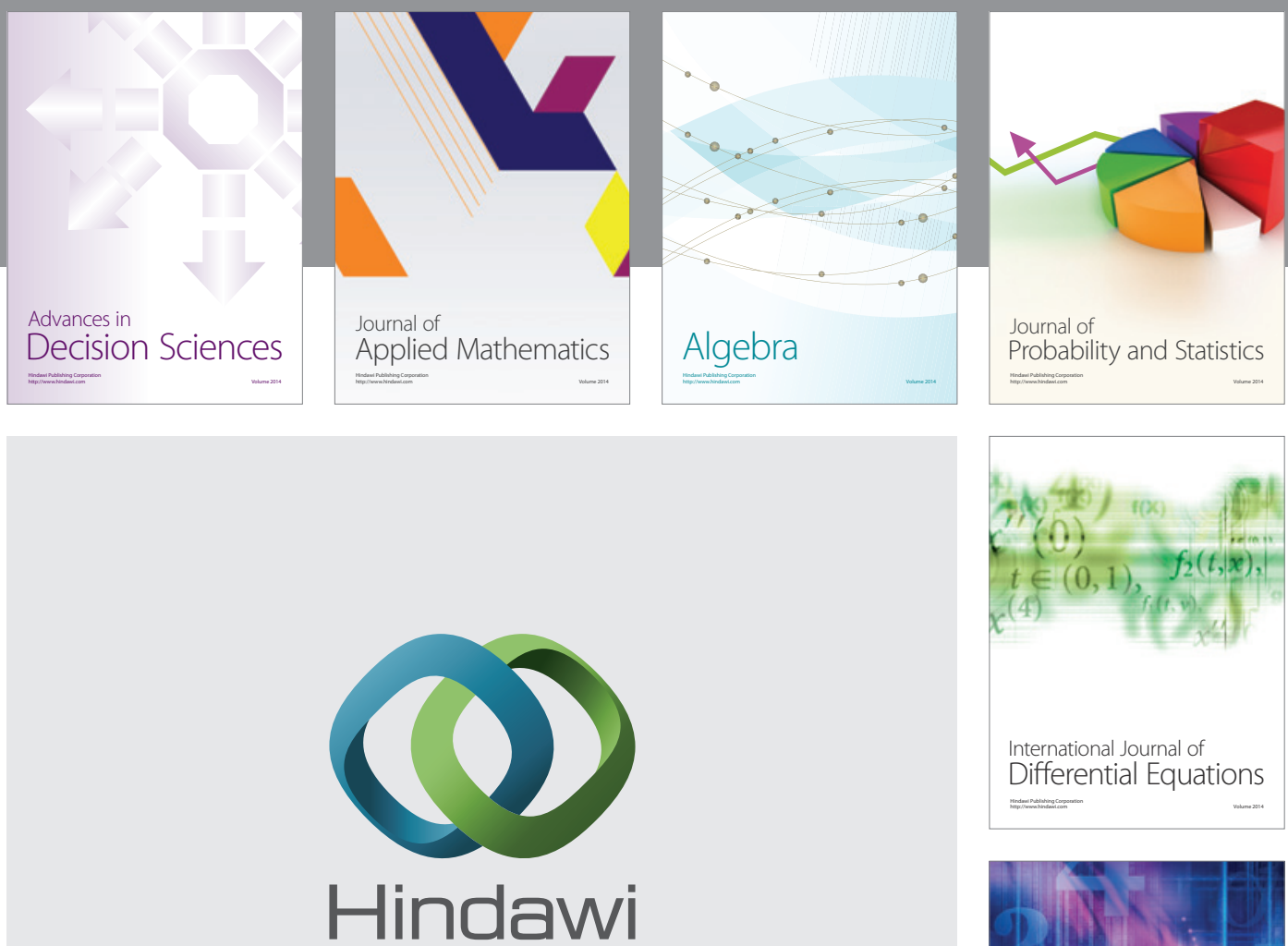

Submit your manuscripts at http://www.hindawi.com
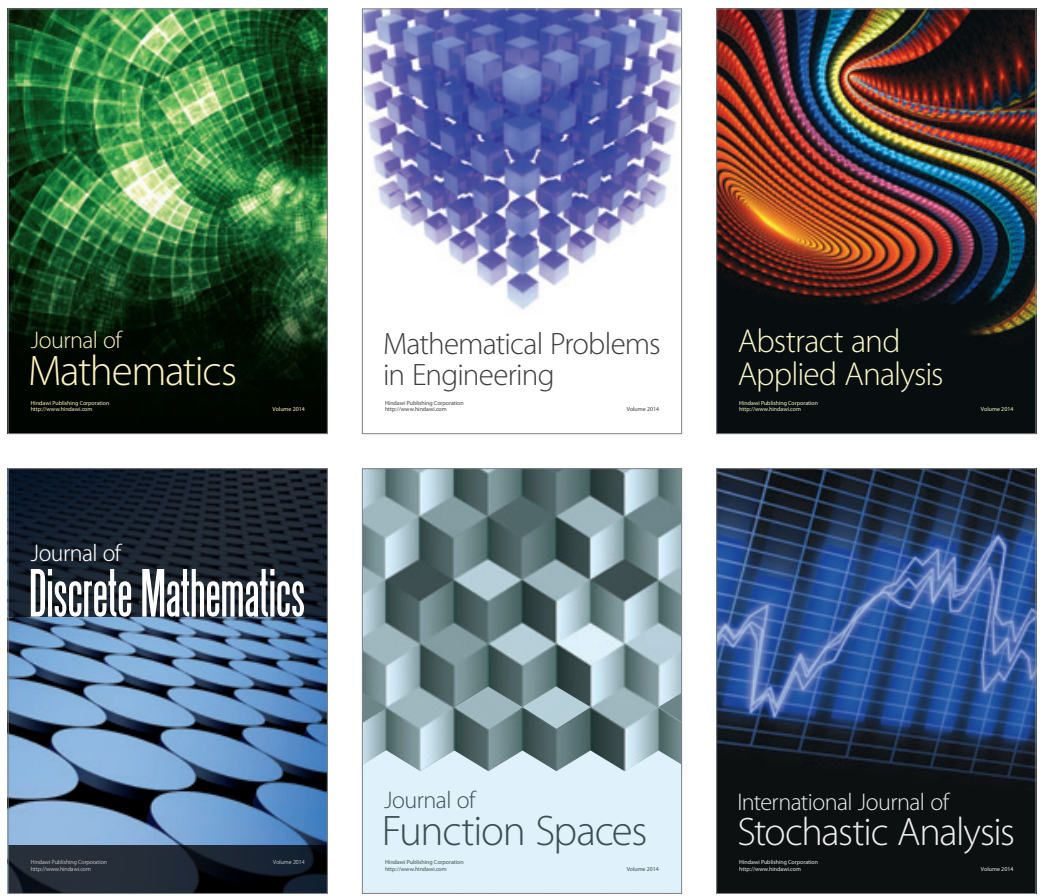

Journal of

Function Spaces

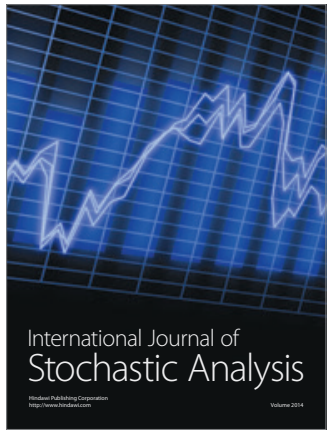

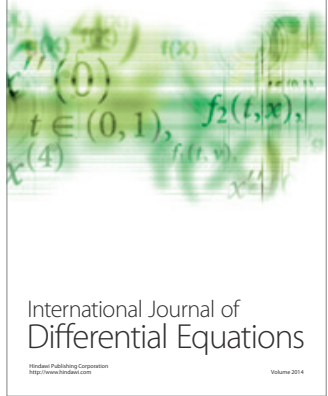
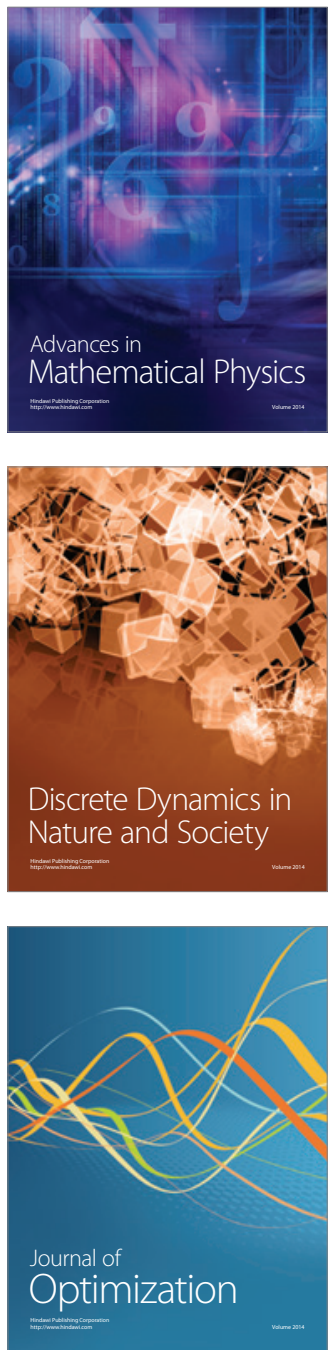